\title{
Low-Cost Interrogation System for Long Period Fiber Gratings as Sensing Devices
}

\author{
P. S. S. dos Santos ${ }^{1,2}$, P.A.S. Jorge ${ }^{1,2}$ José M. M. M. de Almeida ${ }^{1,3}$ L. Coelho $^{1 *}$ \\ ${ }^{1}$ CAP / INESC TEC - Technology and Science and Faculty of Sciences, University of Porto, Porto, Portugal \\ ${ }^{2}$ Dep. of Physics and Astronomy of Faculty of Sciences, Univ. of Porto, 4169-007 Porto, Portugal \\ ${ }^{3}$ Department of Physics, School of Sciences and Technology, University of Trás-os-Montes e Alto Douro, 5001-801 Vila Real, Portugal \\ *Corresponding author: lcoelho@inesctec.pt
}

\begin{abstract}
A system with fiber laser diodes and photodetector replaces the usual bulky and expensive systems for characterization of long period fiber gratings and high correlation is achieved when measuring refractive index, temperature and curvature.

OCIS codes: (060.2370) Fiber Optic Sensors; (050.2770) Gratings; (250.3140) Integrated Optoelectronic Circuits;
\end{abstract}

\section{Introduction}

Long period fiber gratings (LPFG) can be viewed as band-rejection filters with the spectral response tunable by temperature, surrounding refractive index, curvature and strain $[1,2]$ coupled to the well-known advantages of optical fibers including low attenuation losses, immunity to electromagnetic interference and liquid resistance compared with other types of electrical systems makes them very appealing in sensor applications.

This kind of gratings have periods in the range from several tens of microns to some millimeters with a typical spectral rejection band of 10-40 $\mathrm{nm}$ [3]. The shape of this band is close to a Lorentzian function centered at a wavelength $\lambda_{L P F G}$ determined by equation 1 , where $\Lambda$ is the period of the grating and the $n_{\text {core }}$ eff and the $n_{\text {cladding }_{\text {eff }}}$ are the effective refractive index of the core and cladding respectively.

$$
\lambda_{L P F G}=\left[n_{\text {core }_{\text {eff }}}-n_{\text {cladding }_{\text {eff }}}\right] \Lambda=\delta_{\text {neff }} \Lambda
$$

In specific conditions incident light guided by the fundamental mode in the core is coupled to different forward-propagating cladding modes with high order and the transmission spectra shows several rejection bands each one corresponding to a different mode. The sensitivity to the external parameters increases for higher order modes reaching the maximum value at the so called turning point regime [4]. At this point each mode results in two well defined rejection bands with different behaviors allowing to increase the sensitivity to the measurand parameter. The combination with new sensitivity enhanced techniques [5] can induce the development of new sensing systems.

High-resolution interrogation systems for LPFGs have been studied using a wide range of interference techniques as with Mach-Zenders [6], Fabry-Perot cavities [7] and others [8,9]. The standard way of an LPFG characterization consists of a broadband light source connected to the grating and its spectral intensity analyzed in transmission mode by one optical spectral analyzer (OSA). However, these kind of interrogation techniques are not practical for most field sensing applications due to the bulky size, high cost and non-portable so low-cost and portable alternative approaches of interrogation system are desired for many field applications.

Usually the shape of the LPFG rejection band can be fitted by a Lorentzian or a Pseudo-Voigt function, so it can be used to predict the spectral position of the band with just a signal processing. The proposed interrogation method uses a set of narrow and stable fiber laser light sources at the C-band window covering different regions of the LPFG rejection band in study and the power signal is detected with one low-cost InGaAs photodetector. Two different measuring methods can be implemented with prior characterization knowledge of the LPFG resonance band position. The first method implies to keep one laser as close as possible to the rejection band while the other is placed in a spectral region were the power reaches a maximum and stable value acting as a reference. The second method consists on use of both lasers close to the rejection band such that the attenuation affects both lasers intensities allowing higher range and accuracy measurements. It's important to notice that the implementation of these configurations allows the introduction of a second grating to act as a reference to minimize fluctuations due to other parameters.

\section{Material and Methods}

Long period fiber gratings inscribed in single mode fiber (SMF28e, Corning, Inc.), were produced using the plasma-induced electric arc-discharge technique [10]. The period of the LPFG was $400 \mu \mathrm{m}$, a value chosen to obtain the asymmetric $6^{\text {th }}$ order cladding mode resonance $\left(\mathrm{LP}_{1,6}\right)$ around $1550 \mathrm{~nm}$. A visibility value of $\sim 25 \mathrm{~dB}$ for the $\mathrm{LP}_{1,6}$ attenuation band of the bare $\mathrm{LPFG}$ was reached with a sensor length of $45 \pm 5 \mathrm{~mm}$. 
The experimental setup is represented in Figure 1 and is composed by two laser diodes (SPL, Roithner LaserTechnik, Austria) centered at $1529 \mathrm{~nm}$ and $1552 \mathrm{~nm}$ with maximum intensities of $1.56 \mathrm{dBm}$ and $-5.73 \mathrm{dBm}$, respectively. The lasers were connected to a 50:50 fiber coupler and both light signals were sent to the LPFG and connected to a second 50:50 fiber coupler which allows to monitor and compare the output power with the OSA (Yokogawa AQ 6370D) and with one photodiode (EPM605LL, JDSU, EUA) with responsivity of $0.92 \mathrm{~A} / \mathrm{W}$ in the 1500-1600 $\mathrm{nm}$ range, and amplified to output measurable values to a standard voltmeter. Both lasers were controlled with a developed system with a laser driver and a temperature controller to avoid fluctuations in the intensity and spectral drifts.

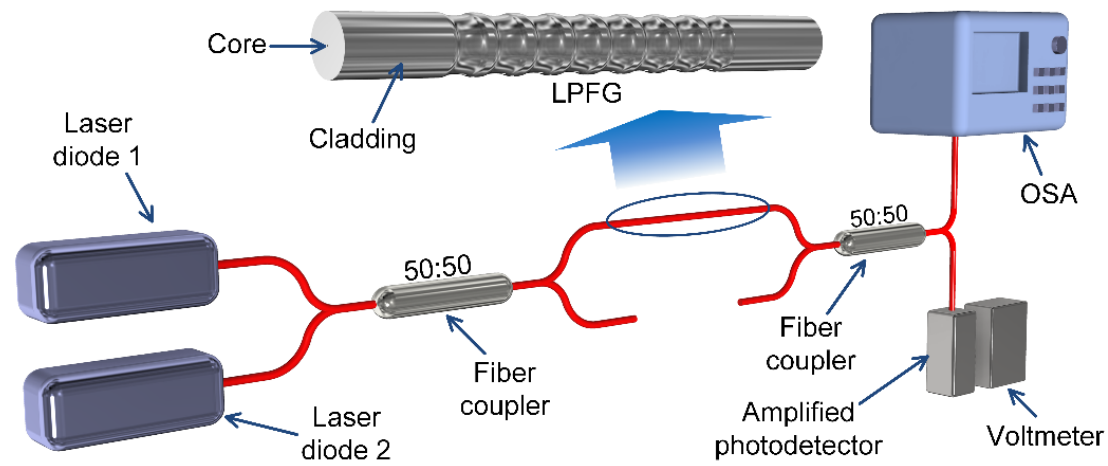

Fig. 1: Laboratorial setup with the LPFG placed between two fiber couplers illuminated with two lasers and detected by an Optical spectrum analyzer and a photodetector.

The setup was applied to the measurements of different parameters as external refractive index, curvature and temperature. In both cases the LPFG was fixed to a precision micrometric positioner to avoid cross measurements. The external refractive index values were obtained using different concentrations of Ethylene glycol mixed with distilled water to obtain values between the range from 1.3333 and 1.4251 . The refractive index was measured with a high-resolution Abbe refractometer (MOD. DR-A1, Atago, Japan). The curvature was estimated through the combination of the length variation between the fixing points and the height variation at the LPFG region. The temperature measurements were performed inside of an incubator (Isotherm, Esco, Singapore) and stretched with a $6 \mathrm{~g}$ mass to avoid bending effect due to the intrinsic silica properties. The resulting LPFG spectra were also record with the OSA in transmission mode by illuminating with a super luminescent diode source (Benchtop SLD model S5FC1550S-A2 - Thorlabs, Germany). This additional data acquired for all the parameters studied is useful for the system calibration and helps the analysis of the grating behavior when changes are applied.

\section{Results}

The results are presented and compared taking in consideration specific parameters, refractive index, curvature and temperature using the same characterization method.

\subsection{External refractive index measurements}

The spectra corresponding to the refractive index variations when illuminating with the laser sources are presented in figure 2. Inset is the LPFG spectra when illuminated with the SLD source and the dashed lines marks the spectral position of the lasers. It's possible to observe that increasing the external RI the rejection band shifts to lower wavelengths and affects the intensity of the laser peaks. The intensity of the laser located at $1529 \mathrm{~nm}$ decreases while the other one increases as expected. Both laser intensities are used to estimate the position of the LPFG band and the consequently the external RI in a wide range of values.

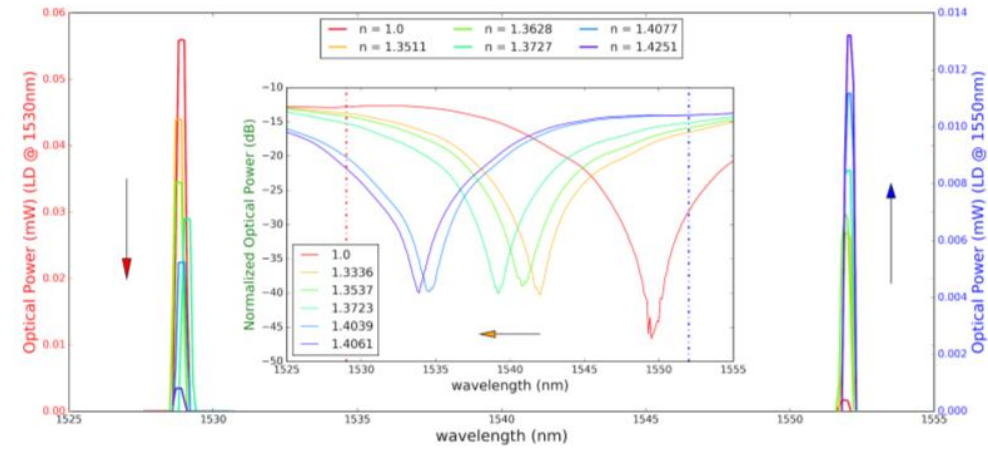

Fig. 2: Optical response of the long period fiber grating by changing the external refractive index when illuminated with the two laser beams. Inset is the spectra when illuminated with a broad band source. 
The results from the photodiode measurements are represented in figure 3 showing a good agreement with the original measured values with calculated correlation coefficients of 0.83 and 0.95 for the laser centered at $1529 \mathrm{~nm}$ and $1552 \mathrm{~nm}$, respectively.
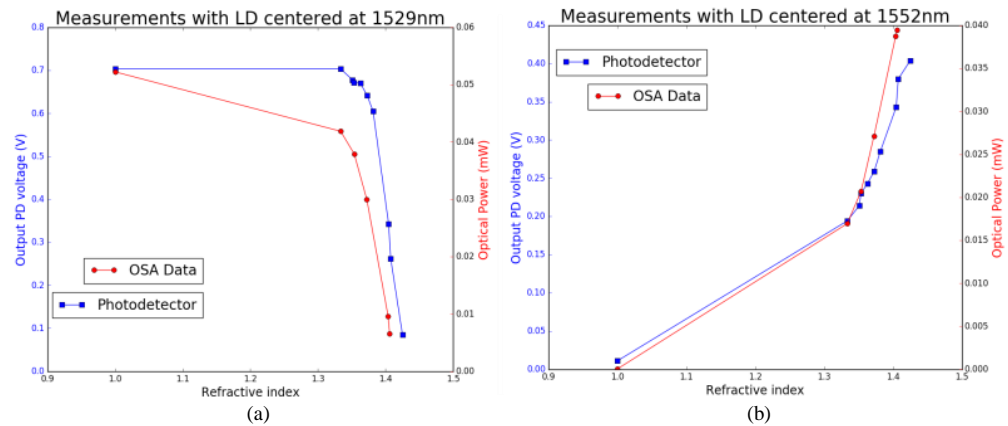

Fig. 3: Comparison between the power measured by the OSA and the voltmeter by changing the external refractive index: (a) laser centered at $1529 \mathrm{~nm}$ and (b) laser centered at $1552 \mathrm{~nm}$

\subsection{Curvature measurements}

The spectral behavior of the rejection band when curvature is applied is slightly different. It is observed in figure 4 (inset) that the band suffers a strong reduction while shifts to higher wavelengths. These effects affect the intensity of the laser located at $1552 \mathrm{~nm}$ while the other located at $1529 \mathrm{~nm}$ only have only some fluctuations in the power intensity detected by the photodetector. The advantage in this case is the fact of having a laser diode for the measurement and another for a reference so any power loss due to non-measurement related external factors will be detected. The results for these measurements are represented in figure 5, the last figure shows remarkably correlation coefficients of 0.99 between the measured values by the expensive OSA and the low-cost voltmeter.

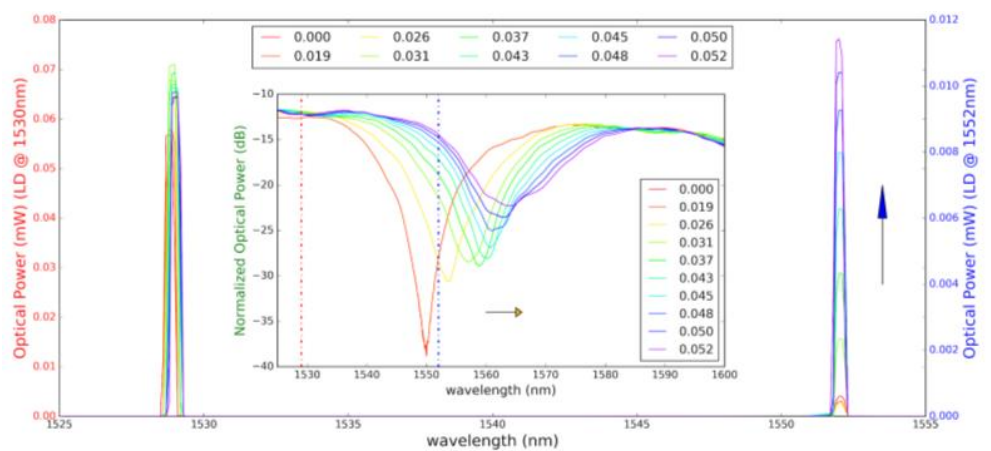

Fig. 4: Optical response of the long period fiber grating by changing the fiber curvature when illuminated with the two laser beams. Inset is the spectra when illuminated with a broad band source.

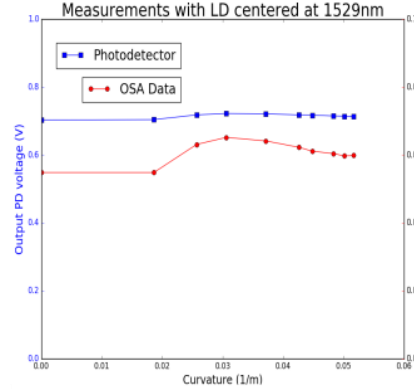

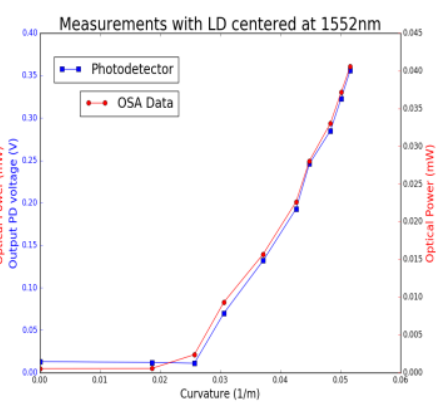

(b)

Fig. 5: Comparison between the power measured by the OSA and the voltmeter for different curvatures: (a) laser centered at $1529 \mathrm{~nm}$ and (b) laser centered at $1552 \mathrm{~nm}$.

\subsection{Temperature variation measurements}

Temperature induced variations in wavelength shift presented in figure 6 shows a thermal sensitivity of $145 \mathrm{pm} /{ }^{\circ} \mathrm{C}$ and with the position of the measurement laser at $1552 \mathrm{~nm}$ the system is able to measure temperatures from $0{ }^{\circ} \mathrm{C}$ to $120^{\circ} \mathrm{C}$. Additional range can be reached by adding a third laser diode positioned at $1570 \mathrm{~nm}$ to reach to temperatures of $220^{\circ} \mathrm{C}$ with the same LPFG. Results are shown in figure 7 . The laser diode located outside the rejection band acts once again as a reference for the measurement signal. 


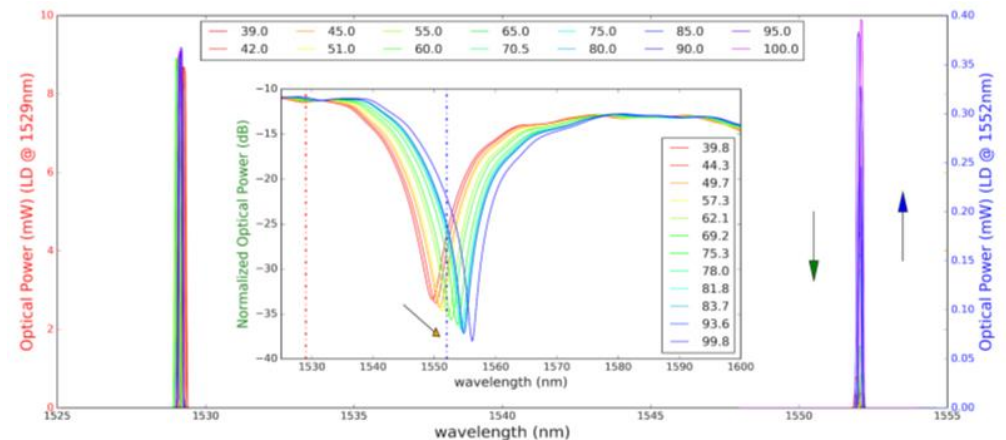

Fig. 6: Optical response of the long period fiber grating by changing the temperature when illuminated with the two laser beams. Inset is the spectra when illuminated with a broad band source.

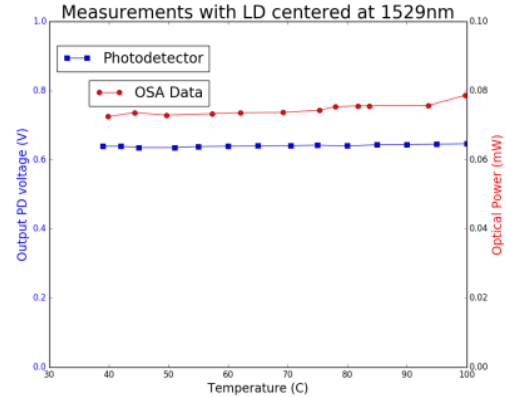

(a)

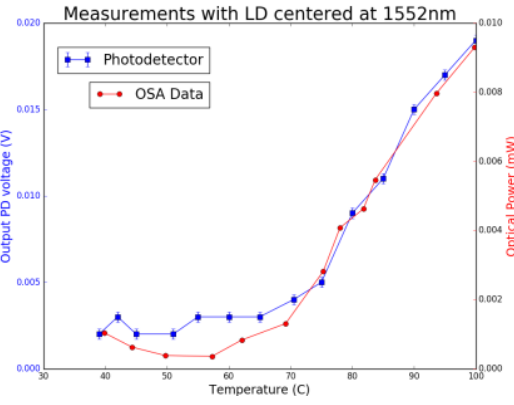

(b)

Fig. 7: Comparison between the power measured by the OSA and the voltmeter for different temperatures: (a) laser centered at 1529 nm and (b) laser centered at $1552 \mathrm{~nm}$.

In this case the sensing laser was placed on the right side of the LPFG band and increasing the temperature the laser intensity decreases to a minimum increasing for higher temperature values.

\section{Conclusions}

A lost-cost system to interrogate the spectral behavior of long period fiber gratings was developed and tested when changing different parameters. The system composed by two laser diodes and one photodetector is used to interrogate long period fiber gratings replacing the usual, bulky and expensive systems. Measurements of sensitivity of refractive index, temperature and curvature were obtained with average correlation coefficient values, for both lasers, of $0.89,0.99$ and 0.95 respectively. The possibility to add more lasers diodes to the system at different wavelengths can improve the measurement accuracy and the range of detection. In addition, the modulation of the lasers diodes accompanied by a dedicated signal processing opens the possibility to scan the rejection band to better estimate the real position in the spectra and consequently the value of the measurand.

\section{Acknowledgments}

This work was financed through the European Regional Development Fund (ERDF) within the project Coral Sustainable Ocean Exploitation: Tools and Sensors/NORTE-01-0145-FEDER-000036."

\section{References}

[1] Vengsarkar, A.M., et al., Long-period fiber gratings as band-rejection filters. Journal of lightwave technology, 1996. 14(1): p. 58-65.

[2] Rego, G., A review of refractometric sensors based on long period fibre gratings. The Scientific World Journal, 2013.2013.

[3] Coelho, L., et al., Fabrication and Characterization of Metal Oxide-Coated Long-Period Fiber Gratings. J. Lightwave Technol., 2016. 34(10): p. 2533--2539.

[4] Del Villar, I., Ultrahigh-sensitivity sensors based on thin-film coated long period gratings with reduced diameter, in transition mode and near the dispersion turning point. Optics express, 2015. 23(7): p. 8389-8398.

[5] Coelho, L., et al., Enhanced refractive index sensing characteristics of optical fibre long period grating coated with titanium dioxide thin films. Sensors and Actuators B-Chemical, 2014. 202: p. 929-934.

[6] Allsop, T., et al., A high sensitivity refractometer based upon a long period grating Mach-Zehnder interferometer. Review of scientific instruments, 2002. 73(4): p. 1702-1705.

[7] Mosquera, L., et al., In-fiber Fabry-Perot refractometer assisted by a long-period grating. Optics letters, 2010. 35(4): p. 613-615.

[8] Xian, L., P. Wang, and H. Li, Power-interrogated and simultaneous measurement of temperature and torsion using paired helical longperiod fiber gratings with opposite helicities. Optics Express, 2014. 22(17): p. 20260-20267.

[9] Jorge, P.A., et al., Fiber optic-based refractive index sensing at INESC Porto. Sensors, 2012. 12(6): p. 8371-8389.

[10] Rego, G., J. Santos, and H. Salgado, Refractive index measurement with long-period gratings arc-induced in pure-silica-core fibres. Optics Communications, 2006. 259(2): p. 598-602. 